\title{
Analysis of active interference on radio station from AC UHV power
}

\author{
ZHANG Jiangong ${ }^{2}$, GAN Zheyuan ${ }^{2}$ and ZHAO Jun and Lu ying ${ }^{2}$ \\ ${ }^{1}$ China Electric Power Research Institute , Wuhan, China \\ ${ }^{2}$ North China Electric Power University, Beijing 102206, China
}

\begin{abstract}
The problems of Electromagnetic Interference in Airborne Intelligence Radar Stations near the AC UHV Transmission Lines are researched in this paper, which is mainly active interference. The calculation method for the interferences is analysed, and then the proper protection distances of the AC UHV transmission line to the radar station are given. The paper analyzed the active interference of AC UHV transmission lines, and focuses on the active interference caused by the line corona on the active radar of nearby airborne intelligence when the transmission line is under normal operation. In the end, this paper proposes the recommended values of active interference protection distance for $1000 \mathrm{kV}$ AC UHV transmission lines and airborne intelligence radar stations.
\end{abstract}

\section{Introduction}

With the rapid development of current science and technology, UHF radio stations(such as analog TV and digital TV broadcasting station, military aviation radars, etc. ), which have strict requirements on the surrounding electromagnetic environment. The solution of passive interference of these stations with UHV transmission lines with large size metal structures has become a research hot spot $[1,2]$.

The air-to-air intelligence radar is the radar which used to search, monitor, identify airborne targets and determine their coordinates and motion parameters, it also known as the air-to-air search radar[3].It is an important electronic technology for acquiring air target information in modern warfare equipment.

The paper analyzed the active interference of AC UHV transmission lines, and focuses on the active interference caused by the line corona on the active radar of nearby airborne intelligence when the transmission line is under normal operation. And then, this paper proposes the recommended values of active interference protection distance for $1000 \mathrm{kV}$ AC UHV transmission lines and airborne intelligence radar stations.

\section{Analysis of active interference of transmission lines and airborne radar stations}

The operating frequency of the radar[3].The operating frequency of the applicable radar has been extended from the high frequency band $(3 \mathrm{MHz}$ to $30 \mathrm{MHz})$ to the millimeter band $(30 \mathrm{GHz}$ to $300 \mathrm{GHz})$. The air-to-air intelligence radar studied in this paper is a commonly used in China. The frequency is $80 \mathrm{MHz}$ to $3000 \mathrm{MHz}$, which can be divided into two frequency bands: $80 \mathrm{MHz}$ to $300 \mathrm{MHz}$ and $300 \mathrm{MHz}$ to $3000 \mathrm{MHz}$. The definition of guard spacing and the guard spacing limit[4] are given in GB13618-1992" Electromagnetic Environment Protection Requirements for Airborne Information Radar Stations". Table 1 shows the spacing between the overhead power lines and the airborne intelligence radar stations at different voltage levels. If it cannot meet the requirements of Table 1 or special circumstances, it can be calculated according to the electromagnetic environment protection criteria of the air intelligence mine station. The interference effects of high-voltage overhead transmission lines and substations considered in the electromagnetic environment protection criteria mainly come from two aspects: First, the high-voltage overhead transmission lines, substations, etc. in the live operation, will produce radio interference; Second, the high-voltage overhead transmission lines, towers and other facilities are used as obstacles, if they are too close to the radar station, it will destroy the requirement of the reflector surface of the radar array, which will cause the deformation of the radar lobe and affect the detection performance. In addition, highvoltage overhead transmission lines, towers and other facilities will also cause a certain shielding angle or electromagnetic wave attenuation of the radar, which may cause the missing radar target or detection distance. According to GB13618-92 "Electromagnetic Environment Protection Requirements for Airborne Information Radar Stations" [4] (hereinafter referred to as "Standard 1") and GJBZ20195-3 "Selection Criteria for Military Ground Radar Positions"[5] (hereinafter referred to as "Standard 2"), the protection spacing for 
radar overhead transmission lines can be calculated separately. The larger one is the final guard spacing value.

Table 1. The protection space between overhead transmission line and intelligence radar station

\begin{tabular}{cccc}
\hline & \multicolumn{2}{c}{ Protective spacing } \\
& & $80 \sim 300 \mathrm{MHz}$ & $300 \sim 3000 \mathrm{MHz}$ \\
\hline \multirow{2}{*}{$\begin{array}{c}\text { High-voltage } \\
\text { overhead }\end{array}$} & $\begin{array}{c}500 \mathrm{kV} \\
\text { transmission lines }\end{array}$ & $1.6 \mathrm{~km}$ & $1.0 \mathrm{~km}$ \\
& $330 \mathrm{kV}$ & $1.2 \mathrm{~km}$ & $0.8 \mathrm{~km}$ \\
& $110 \mathrm{kV}$ & $1.0 \mathrm{~km}$ & $0.7 \mathrm{~km}$ \\
\hline
\end{tabular}

For the radio interference level of High-voltage overhead transmission lines above $30 \mathrm{MHz}$, the above has mainly come from accidental factors such as sparks discharge of iron towers, insulators strings, and metal fittings. At present, there is no effective calculation and prediction method, and only through actual measurement.

The measuring instrument uses SCHWARZBECK $9 \mathrm{k} \sim 3000 \mathrm{MHz}$ receiver, supporting $30 \mathrm{MHz} 300 \mathrm{MHz}$ broadband double-cone antenna and logarithmic period antenna of $200 \mathrm{MHz} 1000 \mathrm{MHz}$ frequency band. The radio interference quasi-peak of the antenna in both the vertical and horizontal polarization directions was measured in the measurement[6].

Since the radio interference level above $30 \mathrm{MHz}$ is very low, the method of comparative measurement under the condition that the test line segment is charged and not charged id adopted. The data measured under the condition that the line segment is not charged is the background radio noise, and by comparison, it is concluded whether the radio interference of the UHV transmission line in this frequency band is significant[78]. Figure 1 shows Radio interference quasi-peak spectrum in the $30 \mathrm{MHz}$ to $1000 \mathrm{MHz}$ band .Figure 2 shows the influence of antenna polarization direction on measuring results.

Radars generally operate in ultrashort waves and above, and are therefore mainly affected by high frequency noise pulses. The mechanism of radar being reduced by noise interference[3] is as fellow: the interference noise from the transmission line enters the radar receiver and is superimposed on the internal noise, reducing the signal-to-noise ratio, causing the radar to reduce the target discovery probability. To maintain the same probability of discovery and false alarm probability, the power of the minimum detectable signal must be increased, but this will reduce the detection rang of the radar.

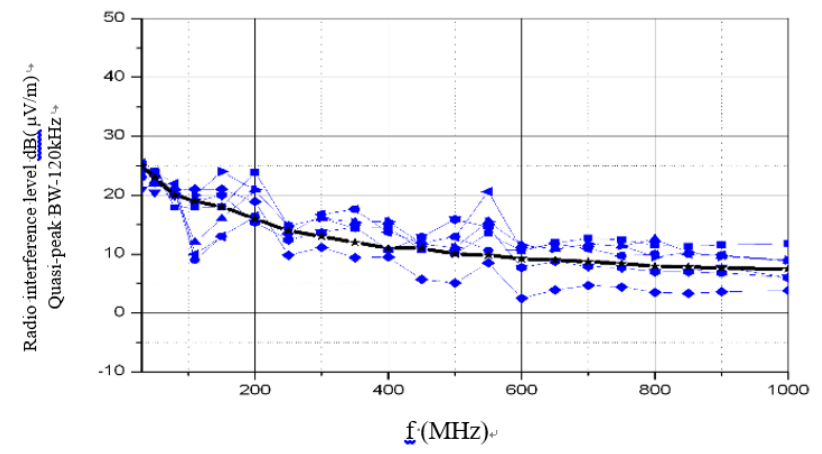

Fig.1 Radio interference quasi-peak spectrum in the 30 $\mathrm{MHz} \sim 1000 \mathrm{MHz}$ band

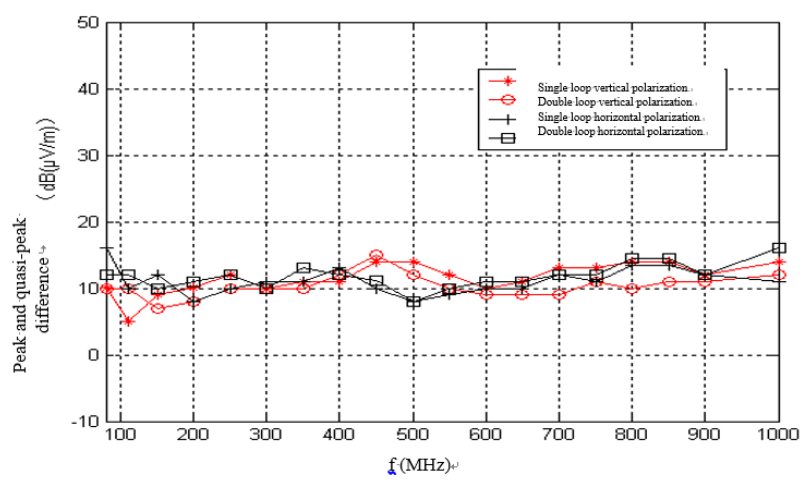

Fig. 2 The influence of antenna polarization direction on measuring results

Figure 3 and 4 summarize two typical comparative measurements. Both measurements were performed in good weather. The measurements did not distinguish between the radio interference levels before and after charging. Therefore, it can only be explained that the UHV line has low radio interference level in this frequency band and is completely submerged in background radio noise[9,10]. Background radio noise is mainly caused by unstable factors such as space radiation and meteorological activities, so that it is presented in an Irregular fluctuations with a small range.

The observation of the iron tower the insulator string, the fittings, etc, of the test line segment by the ultraviolet image also did not clearly reveal the portion where the spark discharge exists(this is an observation made for the specific measured object).

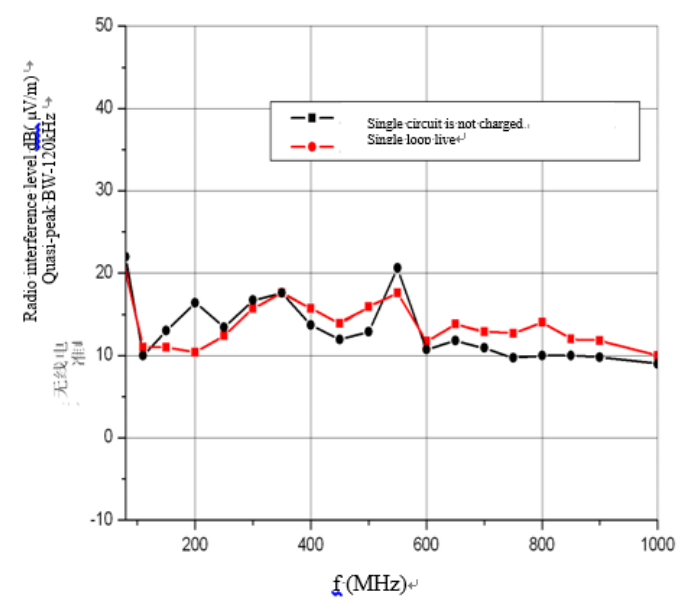

Fig . 3 Comparison I of the radio interference level before and after Charged (vertical polarization) 


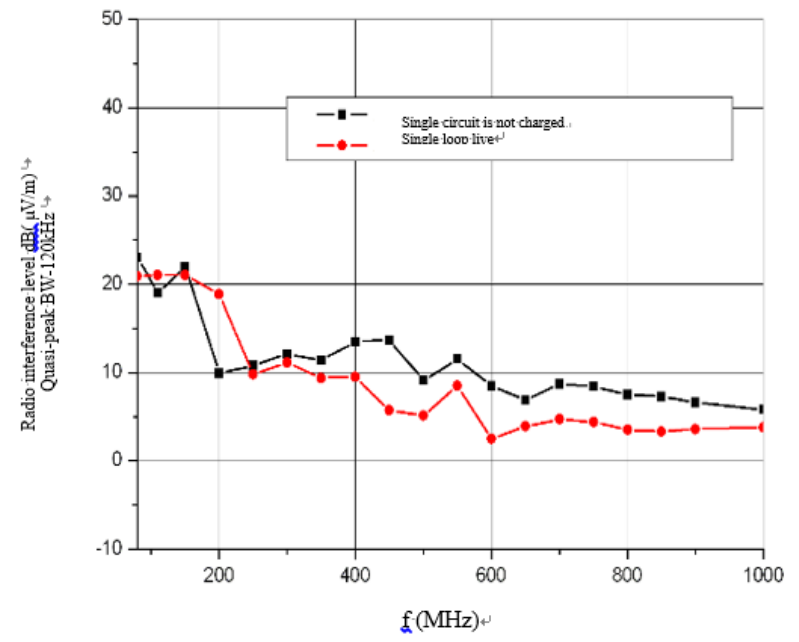

Fig. 4 Comparison II of the radio interference level before and after Charged (vertical polarization)

The measurement results show that the difference between the active radio interference generated by the UHV transmission line and the background noise is very small. Therefore, the impact on radar at working frequencies above $80 \mathrm{MHz}$ is very small. The guard spacing can follow the active guard spacing of the $500 \mathrm{kV}$ transmission line. That is, the guard spacing is $1600 \mathrm{~m}$ when the working frequency is $80 \mathrm{MHz} 300 \mathrm{MHz}$; the guard distance for the radar up to the operating frequency of $300 \mathrm{MHz} 3000 \mathrm{MHz}$ is $1000 \mathrm{~m}$.

\section{Simulation test of the influence of transmission lines on radar}

The test of the influence of obstacles such as UHV transmission line routes and towers on radar detection performance is most ideally carried out on actual circuits. Considering that the transmission line and the tower itself are relatively large, it is difficult to carry out the physical test. Therefore, the" scaling ratio" method is used for the simulation test.

According to Wang Qi, Zhao Jie, and Zhao Zhibin's research on the "passive interference of $800 \mathrm{kV}$ DC transmission lines to the radio stations in the short-wave band", the parameters set in this paper are as follows. The experimental prototype is a "drum type" iron tower. The ratio is $30: 1$, the tower height is about $100 \mathrm{~m}$ $(100 / 30 \mathrm{~m}$ in the test), the transmission line adopts 8 split double loop form, the wire splitting pitch is $400 / 30 \mathrm{~mm}$, and the wire type is $8 \times$ LGJ-500/35 aluminum cable steel reinforced. Stranded wire with a wire radius of $16 / 30 \mathrm{~mm}$ and a pitch of $500 / 30 \mathrm{~m}$. The ground wire is aluminum cable steel reinforced with a grounding resistance of no more than $15 \Omega$. During the test, a 3-pitch 4-tower model was selected for testing.

The transmitting antenna and receiving antenna adopt the double-ridged horn antenna with working frequency band of $1 \sim 18 \mathrm{GHz}$, its average gain is $11.3 \mathrm{~dB}$, the impedance is $50 \Omega$, the maximum power is $300 \mathrm{~W}$, and the peak power is $500 \mathrm{~W}$. The power of the transmitted signal source is E8257D with a maximum output power of $10 \mathrm{dBm}$ and a maximum operating frequency of
40GHz. The receiver uses the E4408B spectrum analyzer and its operating frequency range is $9 \mathrm{kHz} \sim 26.5 \mathrm{GHz}$. The signal source and the transmitting antenna and the spectrum analyzer and the receiving antenna are respectively located in the two sides of the transmission line and the tower, the signal source is connected with the transmitting antenna, and the spectrum analyzer is connected with the receiving antenna ${ }^{[3]}$. The test layout is shown in Figure 5.

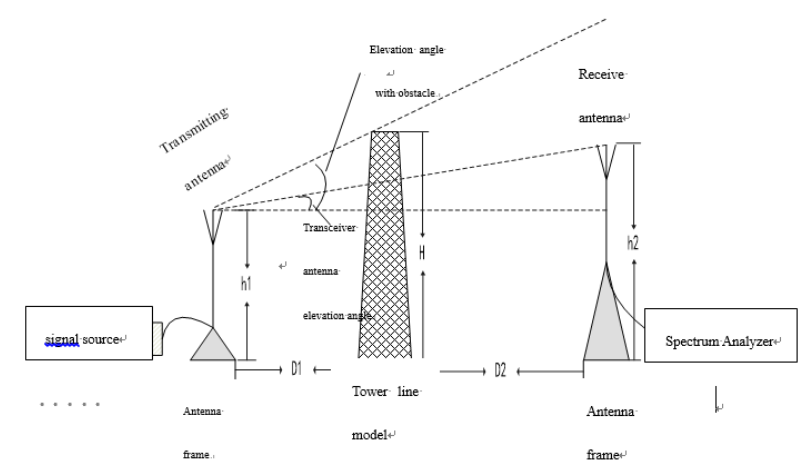

Fig.5 Layout of measurement

Where: $D 1$ distance between the transmitting antenna and the tower line model, units: $\mathrm{m} ; D 2$ distance between the receiving antenna and the tower line model, units: $\mathrm{m} ; h 1$ the height of the transmitting line, units: $\mathrm{m} ; H \longrightarrow$ the height of the tower and line model, units: $\mathrm{m} ;{ }^{h 2}$ _the height of the receiving line, units: $\mathrm{m} ; \alpha$ the elevation angle between the transmitting antenna and the obstruction highest tangent, in degree; $\beta$ - the elevation angle between the connecting line of the transceiver antenna and the horizontal line, in degree.

The main parameters are set as follows: based on the source power and the receiving distance, the distance between the transmitting antenna and the receiving antenna is determined, that is , $(D 1+D 2)$ is $100 \mathrm{~m}$, test frequency selection $3 \mathrm{GHz}, 7 \mathrm{GHz}$ and $12 \mathrm{GHz}$. During the test, follow the steps below.

- Testing the propagation characteristics of electromagnetic waves in free space.

Select the open space, fix the transmitting antenna and receiving antenna at intervals of $100 \mathrm{~m}$, the transmitting antenna frame height is $1.5 \mathrm{~m}$; when the transmitting signal frequency is set to $3 \mathrm{GHz}, 7 \mathrm{GHz}$ and $12 \mathrm{GHz}$ respectively, The corresponding erection height of the receiving antenna is $2.1 \mathrm{~m}, 2.5 \mathrm{~m}, 3 \mathrm{~m}$ and $6 \mathrm{~m}$ respectively. Record the power indicator displayed by the spectrum analyzer;

- Testing the effect of iron tower on the propagation characteristics of electromagnetic waves.

Keep the setting parameters of the spectrum analyzer and the output power of the signal source unchanged, and set the position of the receiving antenna and transmitting antenna unchanged. The tower model is set at $70 \mathrm{~m}, 50 \mathrm{~m}$, $30 \mathrm{~m}$ and $10 \mathrm{~m}$ from the transmitting antenna 
respectively. The corresponding erection height of the receiving antenna is $2.1 \mathrm{~m}, 2.5 \mathrm{~m}, 3 \mathrm{~m}$ and $6 \mathrm{~m}$ respectively. Read the power indicator displayed by the spectrum analyzer;

- Testing the influence of iron towers and transmission lines in the propagation characteristics of electromagnetic waves.

Keep the setting parameters of the spectrum analyzer and the output power of the signal source unchanged, and set the position of the receiving antenna and the transmitting antenna unchanged. Set the assembled iron tower and transmission line models at positions $70 \mathrm{~m}, 50 \mathrm{~m}$, $30 \mathrm{~m}$, and $10 \mathrm{~m}$ away from the transmitting antenna. Record the power indicator displayed by the analyzer.

The test results show that when the transmission frequency is $12 \mathrm{GHz}$, only the tower has a larger attenuation of the received signal than when there is a tower cable. The reason is that when the tower attenuation test is performed, the front of the tower is opposite to the transmitting and receiving antennas, and when there is a tower cable test, the minimum face of the tower is opposite to the transmitting and receiving antennas, resulting in the above results. From the test results, the mainly the influence of the transmission line is the line, and the tower has less influence on the signal transmission. If there is a high-voltage transmission line between the radar and the target, the echo signal received by the radar will pass through the transmission line twice. Since it is difficult to achieve a six-fold increase in the conductivity of the model, the absorption loss of the radar wave by the steel material used in this test model is about 2-3 times larger, so the test results may be stricter.

\section{Conclusion}

In summary, Since the actual measurement shows that the UHV transmission line has a low radio interference level above $80 \mathrm{MHz}$ in the radar operation, it is basically submerged in the background radio interference noise, and even if it is strictly considered, it will not exceed the current $500 \mathrm{kV}$ transmission line. The active interference protection distance of the radar to the UHV transmission line can be implemented according to the requirements of the protection spacing of the $500 \mathrm{kV}$ transmission line in" Standard 1", and the protection distance between the UHV transmission line and the radio station is $1600 \mathrm{~m}$. When using a $1000 \mathrm{kV}$ UHV single-circuit transmission line, the guard spacing is $2400 \mathrm{~m}$. If the height of the radar position is significantly higher than the average height of the transmission line conductor, the protection distance of the meter wave radar to satisfy the effective reflection surface is $2400 \mathrm{~m}$, and the remaining radars can satisfy the active interference protection distance of
$1600 \mathrm{~m}$. However, before determining the protection distance between the $1000 \mathrm{kV}$ UHV transmission line and the air-to-air radar station, it is also necessary to negotiate with the radar station management department. In the specific implementation, if it is difficult to ensure the protection spacing, other areas can be negotiated and resolved in the case that the main responsibility area is satisfied to meet the above requirements.

\section{Acknowledgement}

This work was supported by the Project of SGCC (GY71-16-011).

\section{References}

1. Yin Hui, Zhang Xiaoming, Wang Yantao, et al. Research on 3D Visualization of Electromagnetic Interference for Ultra-high Voltage Transmission Lines[J]. High Voltage Engineering, 40, 12, 2014.

2. Yang Jiawei, Tang Bo, Huang Li, et al. Research on Interference between UHV Power Transmission Lines and Air Defense Surveillance Radar Station[J]. Journal of China Three Gorges University, 2018.

3. Ding Lufei, Geng Fulu, Chen Jianchun, et a. Radar principles[M]. Beijing. Publishing House of Electronics Industry, 2014.

4. GB13618-92. Electromagnetic Environment Protection Requirements for Airborne Radar Stations[S]. Beijing. Standard Press of China, 1992

5. GJBZ20195-3. Selection Criteria for Military Ground Radar Positions[S]. Beijing. Standard Press of China, 1993

6. Gan Zheyuan, Wang Yanhai, Zhang Jiangong, et al. Suppression of HF Passive Interference in Transmission Lines by Using Magnetic Tube[J]. high Voltage Engineering, 43, 5, 2017.

7. Xiang Tao, Hu Yuxian. The electromagnetic Environment Test of the Navigation Station of the Airport in Dazhou, Sichuan Province[J]. China Radio, 11,1, 2017(11)

8. Zhao Jiahao. Causes and Treatment of Wireless Electromagnetic Wave Interference[J]. Electronic Technology and Software Engineering, 21, 1, 2017

9. GB6364-86 Aeronautical Radio navigation Station Electromagnetic Environment Requirements[S]. Beijing. Standard Press of China, 1986

10. GB15707-1995 High-voltage AC Overhead Transmission Line Radio Interference Limit [S]. Beijing. Standard Press of China, 1995 